\title{
The double-edged sword of neutrophilic inflammation in bronchiectasis
}

\author{
Miguel Ángel Martínez-García ${ }^{1}$, Concepción Prados Sánchez ${ }^{2}$ and \\ Rosa María Girón Moreno ${ }^{3}$
}

\begin{abstract}
Affiliations: ${ }^{1}$ Pneumology Service, Polytechnic and University La Fe Hospital, Valencia, Spain. ${ }^{2}$ Pneumology Service, La Paz University Hospital, Madrid, Spain. ${ }^{3}$ Pneumology Service, Institute for Health Research, La Princesa University Hospital, Madrid, Spain.
\end{abstract}

Correspondence: Miguel Ángel Martínez-García, Pneumology Service, Polytechnic and University La Fe Hospital, Bulevar Sur s/n. 46026, Valencia, Spain. E-mail: mianmartinezgarcialagmail.com

@ERSpublications

Inflammation in bronchiectasis: the therapeutic target of the future http://ow.ly/Rlliw

Bronchiectasis is a chronic inflammatory airway disease that is increasingly acquiring a greater epidemiological importance [1]. Its pathogenesis has two main characteristics: bronchial infection and bronchial inflammation. Although bronchial inflammation is mostly (but not always) caused by the bronchial infection, bronchial inflammation is an essential precondition for the development of bronchiectasis. On the cellular level, the inflammation predominantly comprises neutrophils [2]. The main molecules involved in the recruitment of neutrophils to the bronchial mucosa and the pulmonary parenchyma are interleukin (IL)- $1 \beta$, tumour necrosis factor $\alpha$, leukotriene $\beta 4$ and especially some other cytokines from the CXC family, particularly CXCL8 (better known as IL-8) [2, 3]. These cytokines are distinguished by both low- and high-affinity receptors, designated as CXCR1 and CXCR2, respectively, and have been detected not only in neutrophils but also in a wide range of cells with pulmonary expression (endothelial cells, subsets of T-cells, macrophages, monocytes, eosinophils, basophils, epithelial cells, dendritic cells, mast cells, fibroblasts, type II pneumocytes and smooth muscle cells). CXCR1 and CXCR2 have been shown to be involved in the pathogenesis of various inflammatory and fibrotic pulmonary diseases [3, 4]. In humans CXR1 has been particularly associated with neutrophil degranulation and phagocytosis, while CXCR2 has been related to the adhesion and subsequent chemotaxis of these cells on the site of infection [3]. Increases in both the total number and the percentage of neutrophils, as well as the concentration of some neutrophilic proteolytic molecules (basically neutrophilic elastase (NE), myeloperoxidase (MPO) and metalloproteinase (MMP)-9) have been observed in several airway compartments. In addition they have been associated with greater structural changes as they are capable of damaging all the components of the bronchial wall, thereby destroying it and limiting its capacity to repair itself, resulting in the bronchial dilation characteristic of bronchiectasis [5]. All this means that the two key points for controlling the pathophysiological cascade of neutrophilic inflammation are the blockade of the proteolitic molecules produced by the neutrophils themselves, particularly NE (as reported in studies undertaken on bronchiectasis patients [6]), and, secondly, the blockade of CXCR1 and, more specifically, the selective blockade of CXCR2, which reduces bronchial neutrophilic inflammation by preventing the chemotaxis of these cells on the infection site [3]. However, we must remember that neutrophils are also one of the main lines of defence against bacterial infection and other microorganisms found in most bronchiectasis patients, and that bacterial products with a proteolitic capacity play a role in worsening the progression of the disease [2].

Consequently, two of the essential foundations of any treatment of bronchiectasis must be antibiotics and anti-inflammatory drugs. Although there has been significant progress in both respects in recent decades, investigation has tended to focus on improvements in drugs already on the market, as regards dosage

Received: June 182015 | Accepted: June 222015

Conflict of interest: None declared.

Copyright OERS 2015 
(fewer intakes per day), the administration route (inhaled and nebulised antibiotics), increased potency along with reduced side-effects (broncodilators, inhaled steroids and hypertonic saline) and molecules with a proven degree of anti-inflammatory action (macrolides) [7]. However, barely any studies, so far, have seriously investigated any new products with new action mechanisms (particularly in the case of anti-inflammatory drugs) or new pathophysiological routes or pathways. Such research is especially necessary in the field of neutrophilic inflammation, in the light of its relative steroid-resistant condition (corticosteroids are currently the most powerful anti-inflammatory drugs available) and the sub-optimal effect of other anti-inflammatory drugs like macrolides. Accordingly, in the last few years we have witnessed the emergence of a series of molecules designed to impact from the production of neutrophils in the bone marrow to their arrival at the infection site via the bloodstream and the secretion of proteolitic substances. Thus, STOCKLEY et al. [6] recently evaluated the effectiveness and safety of a neutrophilic elastase inhibitor (AZD9668) in bronchiectasis patients and found a significant functional improvement and a trend toward a reduction in inflammatory biomarkers. Furthermore, recent clinical studies have evaluated the efficacy and safety of blockers of CXCR2 receptors in diseases with marked neutrophilic inflammation, such as COPD [8], neutrophilic asthma [9], cystic fibrosis [10] and some interstitial diseases [11], but no study has yet focussed on the effect of these new molecules on bronchiectasis patients.

In this latest issue of the European Respiratory Journal, DE SoyzA et al. [12] analyse, for the first time in the literature, the effect of a new molecule that blocks the CXCR2 receptors (AZD-5069) in a pilot clinical study in patients with bronchiectasis. They conclude that this new molecule significantly reduces the absolute neutrophil cell count in sputum by $69 \%$ and the percentage by $39 \%$ compared with placebo, as well as reducing sputum weight and presenting good tolerance, without any increase in the number or severity of infective exacerbations. Paradoxically, an increase was observed in sputum IL- 6 and GRO- $\alpha$ and serum GRO- $\alpha$, IL-1 $\beta$ and IL-8 concentrations, some of which are important neutrophilic pro-inflammatory mediators. The authors explained this phenomenon as a compensatory mechanism triggered by the reduction in circulating neutrophils.

This study undoubtedly has some limitations, most notably its small sample size, short follow-up period and failure to quantify significant clinical outcomes. However, it is is also true that it has the great virtue of using a methodology with strong scientific evidence (a randomised clinical trial) to analyse, for the first time in bronchiectasis, the safety and efficacy of a very promising new molecule with anti-inflammatory characteristics and a specific action against neutrophils. The study provides a positive response as regards the safety and anti-inflammatory efficacy of this new molecule in neutrophilic inflammation but it opens up many more questions, as well as a hugely interesting field of research into bronchiectasis. It will be necessary to find a balance between a strong anti-inflammatory action (preventing bronchial damage due to inflammation) and an excessive one that weakens the defences against infection (commonly found in bronchiectasis patients, sometimes to a serious degree). This necessity is even more pertinent when we consider that these products could induce neutropenia $[8,13]$ and that CXCR2 has been shown to play a role in the host pulmonary immune response to Pseudomonas [14], Aspergillus [15] and Nocardia [16] infections (all very frequent microorganisms in bronchiectasis). This has led some authors to postulate that any successful approach would require a selective blockade of CXCR2 (the main cause of the chemotaxis of neutrophils) as opposed to CXCR1 (responsible for activation against infections) [3]. It will also be important to make a long-term evaluation of adverse effects (particularly neutropenia, which some of these products are capable of triggering); pharmacological interactions; synergic action with other previously known anti-inflammatory drugs; the patient groups that would most benefit; the effect of smoking on the efficacy of the product; and the variable(s) that need to be monitored to best evaluate efficacy. We must also find explanations for the paradoxical effects observed when these molecules are put to use, such as the increase in some acute-phase reactants and pro-inflammatory molecules [8]. Until all this is achieved, AZD-5069 will occupy a place among molecules that show promise in the management of bronchiectasis but require further studies before any significant clinical impact can be demonstrated. Nevertheless, studies such as that of Soyza et al. [12] do serve to show that after several years of great efforts to raise awareness in the scientific community, bronchiectasis has finally been recognised as a major disease that deserves clinical trials of its own, with specific new products. It is, therefore, to be hoped that neutrophilic anti-inflammatory drugs can follow in the footsteps of other successful randomised clinical trials conducted on bronchiectasis, such as those focussing on inhaled antibiotics and macrolides, as this would offer physicians a potent new tool in the handling of a disease that is sometimes extremely difficult to control.

\section{References}

1 Seitz AE, Olivier KN, Adjemian J, et al. Trends in bronchiectasis among Medicare beneficiaries in the United States, 2000 to 2007. Chest 2012; 142: 432-439.

2 Fuschillo S, De Felice A, Balzano G. Mucosal inflammation in idiopathic bronchiectasis: cellular and molecular mechanisms. Eur Respir J 2008; 31: 396-406. 
3 Chapman RW, Phillips JE, Hipkin RW, et al. CXCR2 antagonists for the treatment of pulmonary disease. Pharmacol Ther 2009; 121: 55-68.

4 Gernez Y, Tirouvanziam R, Chanez P. Neutrophils in chronic inflammatory airway diseases: can we target them and how? Eur Respir J 2010; 35: 467-469.

5 Hoenderdos K, Condliffe A. The neutrophil in chronic obstructive pulmonary disease. Am J Respir Cell Mol Biol 2013; 48: 531-539.

6 Stockley R, De Soyza A, Gunawardena K, et al. Phase II study of a neutrophil elastase inhibitor (AZD9668) in patients with bronchiectasis. Respir Med 2013; 107: 524-533.

$7 \quad$ Chalmers JD, Aliberti S, Blasi F. Management of bronchiectasis in adults. Eur Respir J 2015; 45: 1446-1462.

8 Rennard S, Dale D, Donohue J, et al. CXCR2 antagonist MK-7123. A phase 2 proof-of-concept trial for chronic obstructive pulmonary disease. Am J Respir Crit Care Med 2015; 191: 1001-1011.

9 Nair P, Gaga M, Zervas E, et al. Safety and efficacy of a CXCR2 antagonist in patients with severe asthma and sputum neutrophils: a randomized, placebo-controlled clinical trial. Clin Exp Allergy 2012; 42: 1097-1103.

10 Moss R, Mistry S, Konstan M, et al. Safety and early treatment effects of the CXCR2 antagonist SB-656933 in patients with cystic fibrosis. J Cyst Fibros 2013; 12: 241-248.

11 Russo RC, Guabiraba R, Garcia CC, et al. Role of the chemokine receptor CXCR2 in bleomycin-induced pulmonary inflammation and fibrosis. Am J Respir Cell Mol Biol 2009; 40: 410-421.

12 De Soyza A, Pavord I, Elborn JS, et al. A randomised, placebo-controlled study of the CXCR2 antagonist AZD5069 in bronchiectasis. Eur Respir J 2015; 46: 1021-1032.

13 Holz O, Khalilieh S, Ludwig-Sengpiel A, et al. SCH527123, a novel CXCR2 antagonist, inhibits ozone-induced neutrophilia in healthy subjects. Eur Respir J 2010; 35: 564-570.

14 Tsai WC, Strieter RM, Mehrad B, et al. CXC chemokine receptor CXCR2 is essential for protective innate host response in murine Pseudomonas aeruginosa pneumonia. Infect Immun 2000; 68: 4298-4296.

15 Mehrad B, Strieter RM, Moore TA, et al. CXC chemokine receptor-2 ligands are necessary components of neutrophil-mediated host defense in invasive pulmonary aspergillosis. J Immunol 1999; 163: 6086-6094.

16 Moore TA, Newstead MW, Strieter RM, et al. Bacterial clearance and survival are dependent on CXC chemokine receptor-2 ligands in a murine model of pulmonary Nocardia asteroides infection. J Immunol 2000; 164: 908-915. 